\title{
Slow relaxation in granular compaction
}

\author{
E. Ben-Naim, J. B. Knight, and E. R. Nowak \\ The James Franck Institute, The University of Chicago, Chicago, IL 60637
}

\begin{abstract}
Experimental studies show that the density of a vibrated granular material evolves from a low density initial state into a higher density final steady state. The relaxation towards the final density value follows an inverse logarithmic law. We propose a simple stochastic adsorption-desorption process which captures the essential mechanism underlying this remarkably slow relaxation. As the system approaches its final state, a growing number of beads have to be rearranged to enable a local density increase. In one dimension, this number grows as $N=\rho /(1-\rho)$, and the density increase rate is drastically reduced by a factor $e^{-N}$. Consequently, a logarithmically slow approach to the final state is found $\rho_{\infty}-\rho(t) \cong 1 / \ln t$.
\end{abstract}

PACS numbers: 05.40.+j, 81.20.Ev, 82.65.My

Systems consisting of many macroscopic particles such as sand and powder exhibit complex behavior despite their apparent simplicity [1]. Shaking sand may result in size segregation, rich pattern formation [2,3], or creation of convection rolls [4. Adding a few grains to a sand pile leads to avalanches [5]7. This wealth of phenomena has stimulated analogies to other intriguing physical problems including the motion of flux lines in superconductors, metastability in glassy systems, and surface instabilities in fluids [1].

Despite growing interest, the physical principles underlying sand remain largely unknown. Although individual grains are solid, it is inappropriate to classify their collective properties as entirely solid-like or liquid-like. Conventional thermodynamic theory is not applicable to sand as thermal fluctuations are negligible, $k_{B} T=0$. Most of the experimental and the theoretical studies have focused on spatial inhomogeneities of one sort or another, and much less attention has been given to equally fundamental but simpler situations such as compaction. Granular compaction can be viewed as a model system for nonthermal relaxation in a disordered medium.

Granular compaction is relevant to production, packing, and transportation of a wide array of industrial and agricultural products such as food, grains, chemicals, drugs, and building materials. A granular assembly provides us with a practically uniform system where upon vibration, the well-defined bulk density evolves from a loosely packed mechanically stable initial state into a denser final state (see ref [8] and references therein). The system exhibits no convection, spatial patterns, or oscillatory behavior. The system slowly explores available microscopic configurations, and eventually low-density metastable configurations are eliminated. Several mechanisms have been proposed to explain the kinetics of compaction. The concept of compactivity, which quantifies how far the density is from its maximal value, was argued to play the same role as temperature does in conventional statistical mechanics [10 12]. Such a theory predicts fast exponential relaxation of the density with two relaxation times associated with collective and individual modes [13]. According to another theory, the motion of the voids filling the space between the particles is effectively diffusive, and as a result a power-law relaxation is predicted [14,15]. Although the proposed mechanisms are compelling, their quantitative predictions fail to describe the time dependence observed experimentally [8].

In the compaction experiment of Knight et al [8], monodisperse $2 \mathrm{~mm}$ diameter glass beads are confined to a $1.88 \mathrm{~cm}$ diameter, $1 \mathrm{~m}$ long pyrex tube. The tube is tapped vertically by a vibration exciter. The duration of each pulse is much smaller than the waiting time between different taps so that all of the kinetic energy of the beads is dissipated, and the beads are at rest before the next tap. The column density was measured by a noninvasive capacitive technique. The beads are initially loosely packed with a volume fraction of $0.577 \pm 0.005$. Precautions were taken to prevent humidity (the tube is evacuated), convection, and electrostatic charging. The vibration intensity is parameterized by $\Gamma$, the ratio of the peak acceleration of a tap to $g=9.81 \mathrm{~m} / \mathrm{s}^{2}$. In general, the steady-state density increased monotonically with increasing $\Gamma$. In Fig 1, the time dependence of the packing fraction, $\rho(t)$, is shown for a representative vibration intensity 8]. Shown also is a four-parameter fit $\rho(t)=\rho_{f}-\Delta \rho_{\infty} /[1+B \ln (1+t / \tau)]$. The parameters $\rho_{f}$, $\Delta \rho_{\infty}, B$, and $\tau$ depend only on the acceleration $\Gamma$. This inverse logarithmic form fits the experimental data better than exponential or algebraic laws suggested by the aforementioned compaction theories. Logarithmic relaxation has also been observed in the the decay of the slope of a vibrated sandpile [9].

As the compaction progresses, individual grains move slowly, and when a grain size void is created due to such motion, it is quickly filled by a new grain. New grains can not move into space occupied by other grains. In other words, they interact with their neighbors via a hard core interaction. When the packing fraction is large, voids the size of a particle are rare and a large number of voids have to be rearranged to accommodate an additional particle and a local density increase. Following this line of reasoning, we propose a useful heuristic argument. Let us denote the volume of a particle by $V$ and the pore volume per particle by $V_{0}$. Thus the packing fraction 
is $\rho=V /\left(V+V_{0}\right)$, or alternatively, $V_{0}=V(1-\rho) / \rho$. Assuming that $N$ particles are rearranged in such a way that they contribute their entire free volume to create a particle size void, $N V_{0}=V$. We find $N=\rho /(1-\rho)$. The time associated with such a rearrangement should increase exponentially with $N, T \sim e^{N}$. Consequently, the density increases according to the following rate equation $d \rho / d t \propto e^{-N}=e^{-\rho /(1-\rho)}$. The solution of this equation is given asymptotically by $\rho(t) \cong 1-1 / \ln t$. We made the unrealistic assumption that all of the free volume is available for large void creation. Furthermore, we ignored the structure of the granular assembly, and the fact that the density cannot exceed the close-packed value. These features can be avoided by considering the one-dimensional situation where a void can be uniquely "assigned" to its neighboring particle. Interestingly, the exponential reduction factor agrees with analytic results in $1 \mathrm{D}$.

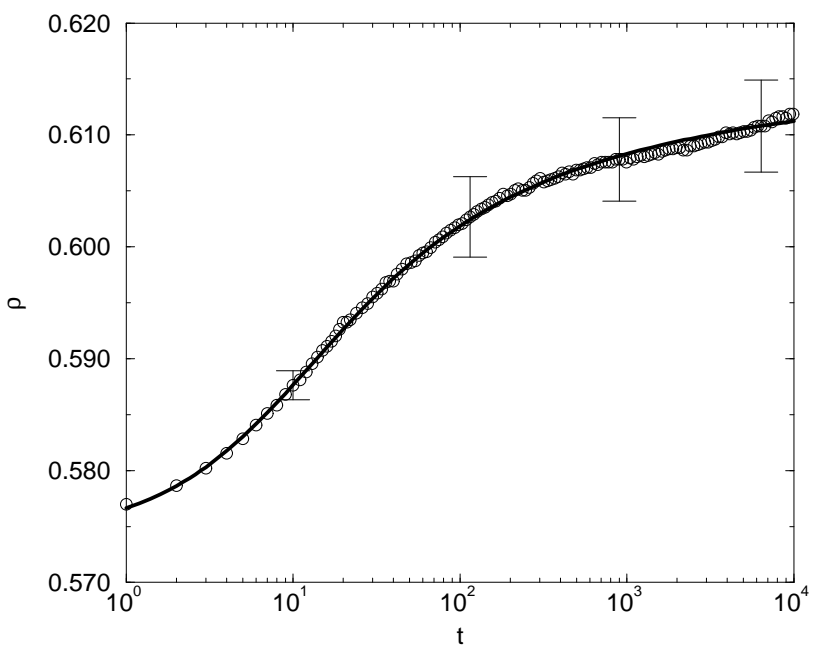

Packing fraction vs. time. Compaction data (o) near the bottom of the tube for acceleration $\Gamma=2.3$. The data represent an average over 5 different runs and the error bars correspond to the rms variations between runs. The solid line is a least square fit to the inverse logarithmic form discussed in the text.

We now propose a simple adsorption-desorption theory for granular compaction. The corresponding stochastic process was previously studied in the context of chemisorption [16 18] and protein binding [19]. The model is defined as follows: Identical spherical particles of unit diameter adsorb uniformly from the bulk to a substrate with rate $k_{+}$and desorb with rate $k_{-}$. In other words, $k_{+}$adsorption attempts are made per unit time per unit length, and similarly, the probability that an adsorbed particle desorbs in an infinitesimal time interval $(t, t+d t)$ is $k_{-} d t$. While the desorption process is unrestricted, the adsorption process is subject to excluded volume constraints, i.e., particles can not adsorb on top of previously adsorbed particles. The attempted adsorption event in Fig. 2 is thus rejected. This stochas- tic process is well-defined in arbitrary dimension. However, we restrict our attention to one-dimension where analytic results are available. This "car-parking" process clearly satisfies detailed balance, and after a sufficiently long time, the system reaches equilibrium. The proposed model can be viewed as a homogeneous one-dimensional gas of hard spheres [20].

To analyze the parking model, it is useful to consider first the simpler lattice version of the process where particles occupy a single lattice site. In this case the density, $\rho$, satisfies the Langmuir mean-field equation $d \rho / d t=k_{+}(1-\rho)-k_{-} \rho$. The gain term is proportional to the fraction of unoccupied space, while the loss term is proportional to the density itself. The steady-state density, $\rho_{\infty}$, which is obtained by imposing $d \rho / d t=0$, depends on the rate ratio $k \equiv k_{+} / k_{-}$only, $\rho_{\infty}=k /(1+k)$. The system relaxes exponentially towards its final state, $\rho_{\infty}-\rho(t)=\left(\rho_{\infty}-\rho_{0}\right) e^{-t / \tau}$, with $\rho_{0}$ the initial density. The relaxation rate is simply a sum of the adsorption rate and the desorption rate $\tau^{-1}=k_{+}+k_{-}$.

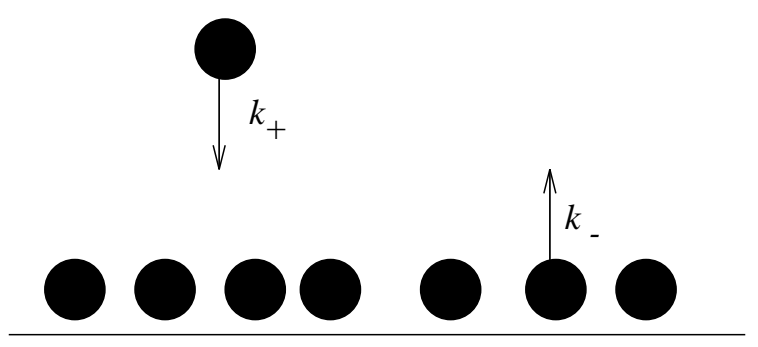

Fig 2 The adsorption-desorption process.

On the continuum, however, only a fraction of the empty space is available for adsorption. It was previously shown that in equilibrium the probability $s(\rho)$ that an adsorption event is successful equals $e^{-\rho /(1-\rho)}[18$. This so-called "sticking coefficient" equals unity in the low density limit, and the above mean-field treatment holds. On the other hand, in the high density limit this coefficient vanishes exponentially, $s(\rho) \propto e^{-1 /(1-\rho)}$, as $\rho \rightarrow 1$. The excluded volume interaction effectively reduces the adsorption rate, $k_{+} \rightarrow k_{+}(\rho)=k_{+} s(\rho)$. Thus, a modified Langmuir equation can be written

$$
\frac{d \rho}{d t}=k_{+}(1-\rho) e^{-\rho /(1-\rho)}-k_{-} \rho .
$$

This evolution equation was constructed to give the exact equilibrium density, and is reasonable as long as the system is sufficiently close to equilibrium.

Using Eq. (17), the equilibrium density is obtained from the following transcendental equation, $\alpha e^{\alpha}=k$, with $\alpha=\rho_{\infty} /\left(1-\rho_{\infty}\right)$. We find the following leading behavior in the two limiting cases

$$
\rho_{\infty}(k) \cong \begin{cases}k & k \ll 1 ; \\ 1-(\ln k)^{-1} & k \gg 1 .\end{cases}
$$


While the mean-field linear relation is recovered in the dilute limit, the dense limit is characterized by a logarithmic cusp. By contrast, mean-field predicts a powerlaw dependence of the density $\rho_{\infty} \cong 1-1 / k$, for $k \gg 1$. The effect of the volume exclusion constraint is striking, a huge adsorption to desorption rate ratio, $k \cong 10^{9}$, is necessary to achieve a 0.95 steady-state occupancy. It is also interesting to consider the equilibrium void distribution. The density of voids of size $x$ is exponential $P_{\infty}(x)=\beta e^{-\alpha x}$, with $\beta=\rho_{\infty}^{2} /\left(1-\rho_{\infty}\right)$ and the previously defined $\alpha[18]$. Indeed, the sticking coefficient is proportional to the density of voids larger than a particle size, $s(\rho) \propto \int_{1}^{\infty} d x P_{\infty}(x)$.

We now focus on the relaxation properties of the system. The granular compaction process corresponds to the high density limit, and we thus focus on the desorption-controlled case, $k \gg 1$. Hence, let us fix $k_{+}$ and consider the limit $k_{-} \rightarrow 0$ of Eq. (11). The early time behavior is dominated by adsorption and can be obtained by neglecting the desorption term. For simplicity, we consider a vanishing initial concentration. For sufficiently early times, $t \ll 1 / k_{+}$, the density grows linearly in time, $\rho(t) \cong k_{+} t$. At the later stages of the process, $t \gg 1 / k_{+}$, the system approaches complete coverage, $\rho_{\infty}=1$, according to [18,21]

$$
\rho(t) \cong \rho_{\infty}-\frac{1}{\ln \left(k_{+} t\right)}
$$

This is confirmed by numerical simulations in one dimension. Use of Eq. (11) is justified a posteriori since the system evolves slowly and has enough time to equilibrate. The inverse logarithmic behavior is simply a reflection of the exponentially suppressed adsorption in the dense limit. In writing Eq. (3) we neglected higher order logarithmic corrections such as $\ln \ln \left(k_{+} t\right)$. We conclude that the excluded volume interaction gives rise to a slow relaxation.

Eq. (3) holds indefinitely only for the truly irreversible limit of the parking process, i.e., for $k=\infty$. For large but finite rate ratios, the final density is given by Eq. (2). As the density approaches this steady state value, the loss term becomes significant and should be taken into account. The crossover time between the two different relaxation regimes, $t_{0}$, can be conveniently estimated by equating the time dependent density of Eq. (3) with the equilibrium density of Eq.(2) $1-1 / \ln \left(k_{+} t_{0}\right)=$ $1-1 / \ln \left(k_{+} / k_{-}\right)$, and as a result $t_{0} \cong 1 / k_{-}$. For $t \gg t_{0}$, the loss term is no longer negligible. By computing how a small perturbation from the steady state decays with time, an exponential relaxation towards the steady state is found $\left|\rho_{\infty}-\rho(t)\right| \propto e^{-t / \tau}$ for $t \gg t_{0}$. The relaxation time is indeed $t_{0}$, however, an additional logarithmic correction occurs, $\tau=t_{0}\left(1-\rho_{\infty}\right)^{2} \simeq t_{0} /(\ln k)^{2}$. The above results can be simply understood: the early time behavior of the system follows the irreversible limit of $k_{-}=0$. Once the system is sufficiently close to the steady-state, the density relaxes exponentially to its final value (Fig 3).
Hence, two relaxation curves with sufficiently large final densities are indistinguishable over a significant temporal range.

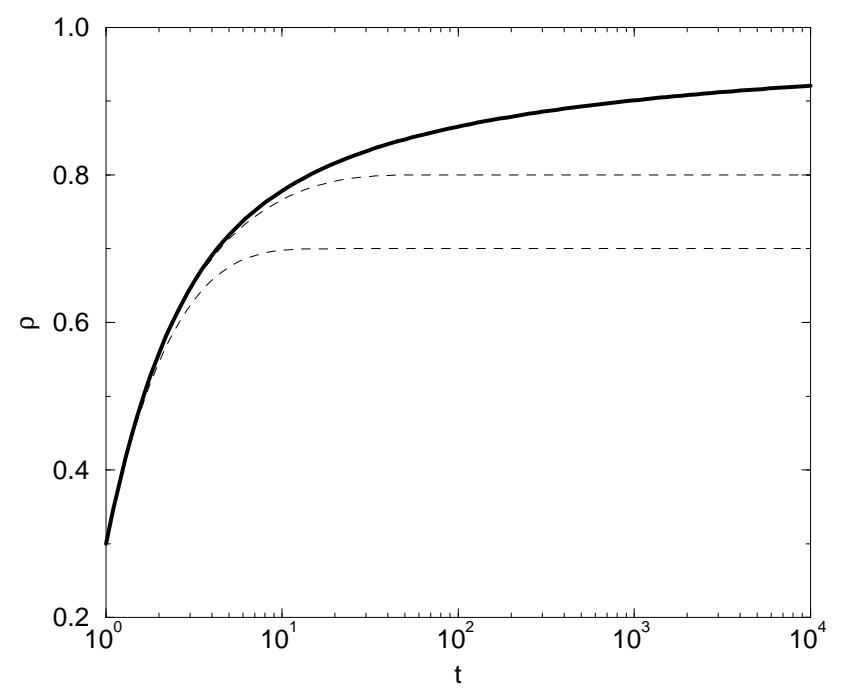

Fig 3 The coverage versus time. Shown are solutions of Eq. (1) with $k_{+}=1$. The upper curve corresponds to the irreversible limit $\rho_{\infty}=1$, while the lower two curves represent reversible parking with equilibrium densities of $\rho_{\infty}=0.7$ and 0.8 respectively.

In the desorption-controlled limit, the void left by a desorbed particle is quickly filled by an adsorbed particle. Hence, the desorption mechanism can be seen as playing a similar role to diffusion of particles. Indeed, theoretical studies show that adsorption processes where adsorbed particles diffuse rather than desorb give rise to the same density relaxation as in Eq. (3) [22]. Our treatment was restricted to one-dimensional processes, but we expect that the results hold in higher-dimension as well. Hence, the above relaxation is rather robust and insensitive to many microscopic details.

The inverse logarithmic density relaxation towards the steady-state is the same one observed for granular compaction (see Fig 1). Furthermore, the experimentally observed relaxation curves which correspond to large compaction, $\Gamma>2.7$, are indistinguishable over the observed time range consistent with the above theory. The largest packing fraction observed in the experiment are well below the maximal close-pack value in $3 \mathrm{D}$. Thus, according to the parking theory the logarithmic relaxation regime may be followed by an exponential relaxation. There exists a maximal time scale which characterizes the temporal behavior. Such a prediction is consistent with the experimental observation that steady state is achieved within a finite time. Finally, once the system reaches its final state, density fluctuations can be measured and compared with Monte Carlo simulations of the parking process. Our preliminary results indicate that measured and simulated Power Spectrum Density (PSD) of the density fluctuations are strikingly similar [24]. The high 
frequency limit is Lorentzian $\left(f^{-2}\right)$, while the low density limit is white-noise $\left(f^{0}\right)$. The intermediate regime is approximately a powerlaw $f^{-\alpha}$, with $0.7<\alpha<1.4$.

In a realistic granular material, an individual particle can not penetrate its neighbors, but it also must be in contact with several other particles. Our model properly accounts for the hard core repulsion, but it ignores mechanical stability. We argue that in the long time limit mechanical stability can not play a significant role in determining the motion of individual grains during the compaction process. Instead, the motion is limited primarily by the presence of other beads. The tradeoff, however, is that such a simple theory can not make predictions about the final density. Nevertheless, it does elucidate the leading mechanism in granular compaction. It will be very useful to try and incorporate the important distinction between total void space and the available void space into the recent theories concerning compaction and packing 10,15,23.

In conclusion, we have studied theoretically density relaxation towards steady state in granular compaction using a microscopic model in one dimension. Due to volume exclusion, exponentially growing time scales are associated with cooperative motion of grains. As a result the approach towards the steady state is an inverse logarithmic one. Since the argument leading to the logarithmic relaxation is a general one, the results should hold in a large class of physical situations, for example when the shaking is horizontal rather the vertical or when the grains are aspherical. Interestingly, the same mechanism is responsible for the long time necessary for packing grains in a bowl, parking a car in a busy street, or even entering a crowded room.

We are grateful to H. M. Jaeger and S. R. Nagel for many stimulating discussions. We also thank P. Dimon, H. J. Herrmann, D. C. Hong, L. P. Kadanoff, P. L. Krapivsky, J. F. Marko, A. Mehta, T. A. Witten for useful discussions. This work was supported by The MRSEC Program of the National Science Foundation under Award Number DMR-9400379, and by the Department of Energy Grant No. DE-FG02-92ER25119. We also wish to acknowledge support from NSF grant \#92-08527 (EB) and NSF (JBK).
[1] H. Jaeger and S. Nagel, Science 255, 1513 (1992). R. P. Behringer, Nonlinear Science Today 3, 1 (1993).

[2] P. Evesque and J. Rajchenbach, Phys. Rev. Lett. 62, 44 (1989).

[3] F. Melo, P. B. Umbanhowar, and H. L. Swinney, Phys. Rev. Lett. 75, 21 (1995).

[4] E. E. Ehrichs, H. M. Jaeger, and S. R. Nagel, Science 267 5204, (1995).

[5] P. Bak, C. Tang, and K. Wiesenfeld, Phys. Rev. Lett. 59, 381 (1987).

[6] H. Jaeger, C.H.Liu, and S. Nagel, Phys. Rev. Lett. 62, 40 (1989).

[7] V. Frette, K. Christianse, and P. Meakin, Nature, 379, 6560 (1996).

[8] J. B. Knight, C. G. Fandrich, C. N. Lau, H. M. Jaeger, and S. R. Nagel, Phys Rev. E 513957 (1995).

[9] H. M. Jaeger, C. H. Liu, and S. R. Nagel, Phys. Rev. Lett. 62, 40 1989). T. A. Duke, G. C. Barker, and A. Mehta, Europhys. Lett. 13, 19 (1990).

[10] S. F. Edwards in Granular Matter, An Interdisciplinary Approach, edited by A. Mehta (Springer-Verlag, New York, 1994).

[11] A. Mehta in Granular Matter, An Interdisciplinary Approach, edited by A. Mehta (Springer-Verlag, New York, 1994).

[12] A. Mehta and S. F. Edwards, Physica A 157, 1093 (1989).

[13] G. C. Barker and Anita Mehta, Phys. Rev. E 47, 184 (1993).

[14] H. S. Caram and D. C. Hong, Phys. Rev. Lett. 67, 828 (1991).

[15] D. C. Hong, S. Yue, J. K. Rudra, M. Y. Choi, and Y. W. Kim, Phys. Rev. E 50, 4123 (1994).

[16] J. W. Evans, Rev. Mod. Phys. 65, 1281 (1993).

[17] R. B. Stinchcombe, M. D. Grynberg, and M. Barma, Phys. Rev. E 47, 4018 (1993).

[18] P. L. Krapivsky and E. Ben-Naim, Jour. Chem. Phys. 100, 6778 (1994).

[19] J. D. McGhee and P. H. von Hippel, J. Mol. Bio 86, 469 (1974).

[20] L. Tonks, Phys. Rev. 50, 955 (1936).

[21] X. Jin, G. Tarjus, and J. Talbot J. Phys. A 27 L195 (1994).

[22] V. Privman and M. Barma, Jour. Chem. Phys. 97, 6714 (1992).

[23] A. Coniglio, H. Herrmann, cond-mat/9602062 (preprint). S. J. Lintz, (preprint).

[24] E. R. Nowak, J. B. Knight, E. Ben-Naim, H. M. Jaeger, and S. R. Nagel, (in preparation). 